\title{
OVARIAN ABSCESS RUPTURE AFTER OOCYTE RETRIEVAL IN A PATIENT WITH ENDOMETRIOMA: CASE REPORT
}

\author{
Nikoleta G. TABAKOVA ${ }^{1,2 \bowtie}$, Emil G. KOVACHEV ${ }^{1,2}$, Vilislava R. IVANOVA ${ }^{1,2}$, \\ Tsvetomir E. KACHOVSKI ${ }^{1,2}$, Dimitar D. IVANOV ${ }^{1,2}$ \\ ${ }^{1}$ Department of Obstetrics and Gynaecology, Medical University Varna, Varna, Bulgaria \\ 2 Obstetrics and Gynaecology Hospital SBAGAL Varna, Varna, Bulgaria
}

Received 06 Jan 2021, Accepted 16 Febr 2021

https://doi.org/10.31688/ABMU.2021.56.1.15

\begin{abstract}
Introduction. Pelvic abscess rupture is a rare complication following in-vitro fertilization procedures.

Case presentation. We describe an unusual case of ovarian abscess rupture following transvaginal oocyte retrieval in a 31-year-old female with a previous endometrioma. The patient presented 17 days after ultrasound-guided oocyte retrieval and 12 days after embryo transfer in our emergency room, with acute lower abdominal pain, septic fever and malaise. Laparoscopic surgery was considered after failure of triple intravenous antibiotic combination.

Conclusions. Managing patients undergoing infertility treatment with this condition appears to be difficult due to the need of aggressive antibiotic and surgical treatment, which increases the risk of spontaneous abortion after embryo transfer.
\end{abstract}

Keywords: embryo transfer, endometrioma, in-vitro fertilization, oocyte retrieval, ovarian abscess.

\section{Résumé}

Rupture de l'abcès ovarien après la récupération d'oocyte chez un patient avec endométriome: rapport de cas

Introduction. La rupture d'un abcès pelvien est une complication rare après des procédés de fécondation in vitro.

Présentation du cas. Nous décrivons un cas inhabituel de rupture d'abcès ovarien après prélèvement d'ovocytes transvaginal chez une femme de 31 ans avec un endométriome antérieur. La patiente s'est présentée dans notre salle d'urgence, 17 jours après le prélèvement d'ovocytes guidé par échographie et 12 jours après le transfert d'embryon, présentant des douleurs abdominales basses aiguës, une fièvre septique et un malaise. Une chirurgie laparoscopique a été envisagée après l'échec d'une triple combinaison d'antibiotiques par voie intraveineuse.

Conclusions. L'approche des patients présentant cette condition par un traitement contre l'infertilité semble être difficile en raison de la nécessité d'un traitement antibiotique et chirurgical agressif qui augmente le 
risque d'avortement spontané dans les cas ayant subi un transfert d'embryon.

Mots-clés: transfert d'embryon, endométriome, fécondation in vitro, prélèvement d'ovocytes, abcès ovarien.

\section{INTRODUCTION}

Ovarian abscess rupture certainly is an uncommon complication following in-vitro fertilization (IVF) procedures. The risk factors that may contribute to the development of the condition following oocyte retrieval are the presence of endometriomas and/or hydrosalpinx $x^{1}$. The case who will be presented suggests the importance to recognize these patients preconceptionally and to minimize their risk by surgical treatment before applying any assisted reproductive techniques. On the contrary, because of the lack of prospective studies and evidence data based only on case reports, there is still a debate in the reproductive field whether or not to recommend the surgical treatment before undergoing IVF cycles in these patients ${ }^{2-5}$.

\section{Case presentation}

A 31-year-old white female, gravida 2, para 1, presented on November the $25^{\text {th }} 2020$, to the emergency room for lower abdominal pain, septic fever and malaise for 10 days. At the start of the infertility treatment, in November 2020, the patient was known with endometrioma in the right ovary, $5 \mathrm{~cm}$ in diameter, diagnosed on its typical ultrasound appearance. By protocol, she received ultrasound-guided transvaginal oocyte retrieval (TVOR) and embryo transfer. During TVOR, an accidental puncture of the endometriotic cyst was suspected. The patient was given prophylactic antibiotics. Despite the antibiotic treatment, two days later she experienced lower abdominal discomfort and fever. Ten days after the onset of symptoms, the patient presented to our emergency room with severe right lower quadrant abdominal pain and nausea. The abdominal palpation revealed marked generalized tenderness, rebound tenderness and guarding. The pelvic examination showed severe bilateral adnexal tenderness to palpation, right greater than left. On admission, she had a temperature of $38.4^{\circ} \mathrm{C}$. The pelvic ultrasound (Figures $1,2,3,4$ ) depicted a multilocular adnexal mass of $8 \mathrm{~cm}$ in diameter, with heterogeneously echogenicity of the inner portions and irregular thick walls in the right adnexal region, with a moderate amount of free fluid in the Douglas pouch and an enlarged retroverted uterus. The differential diagnosis included torsion of the endometriotic cyst or rupture of ovarian abscess due to an inflammation of the endometrioma following TVOR. The patient was started on triple antibiotic therapy with Metronidazole, Gentamicin and Ceftriaxone.

The patient's condition aggravated after admission. Six hours after admission, her abdominal pain increased in severity and the blood pressure dropped to $80 / 40 \mathrm{mmHg}$, pulse rate accelerated to $130 / \mathrm{min}$, and the temperature raised up to $39.5^{\circ} \mathrm{C}$. Laboratory findings were consistent with the presumptive diagnosis of intraabdominal ovarian abscess rupture. A routine blood test showed that the white blood cell count was $16.3 \times 10^{9} / \mathrm{L}$, with $81.1 \%$ granulocytes. The C-reactive protein was $266 \mathrm{mg} / \mathrm{L}$. A decision for emergent exploratory laparoscopy was made. From the rectouterine space $\sim 300 \mathrm{~mL}$ of thick, brownish fluid was aspirated. The left adnexa seemed only hyperaemic and oedematous. A right adnexal mass of approximately $10 \mathrm{~cm} \times 8 \mathrm{~cm} \times 8 \mathrm{~cm}$ in diameter was separated from adherent tissues. The mass arose from the right ovary, and normal ovarian tissue was missing. Right laparoscopic salpingo-oophorectomy was performed. Pelvic drains were left in situ. A culture of vaginal discharge on admission showed no bacterial growth. The histopathological examination showed tubal ovarian abscess. The triple combination of intravenous antibiotics was continued till discharge. Oral antibiotics were prescribed for another 7 days. The pelvic drain was removed on the $3^{\text {rd }}$ day postoperatively. The patient was discharged on the $4^{\text {th }}$ day post- surgery, afebrile and in stable condition.

\section{Discussion}

Endometriosis occurs in 25\% to $50 \%$ of women with infertility ${ }^{6}$. In vitro fertilization is an effective treatment for these women, and although pelvic abscess is a rare complication of oocyte retrieval $(0.3-0.4 \%)$, endometriosis is a risk factor ${ }^{7,8}$. The proposed pathophysiological mechanism is the bloody content of the endometrioma, that may serve as a good culture medium for bacteria and facilitate the spread of infection'. The pseudo-capsule of the endometriotic cyst may prevent antibiotics from overcoming bacteria. Endometriosis may increase the risk of 


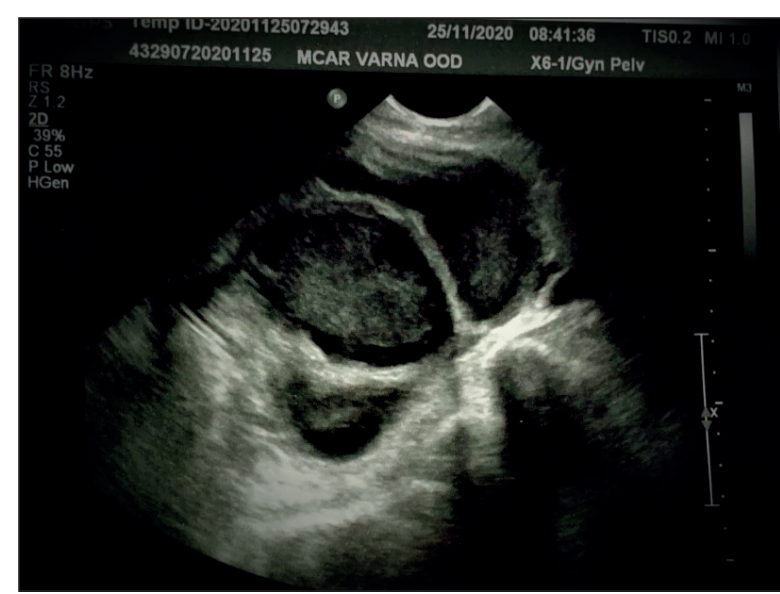

Figure 1. Ultrasonography - a multilocular adnexal mass, $8 \mathrm{~cm}$ in diameter, with heterogeneous echogenicity of the inner portions and irregular thick walls in the right adnexal region.

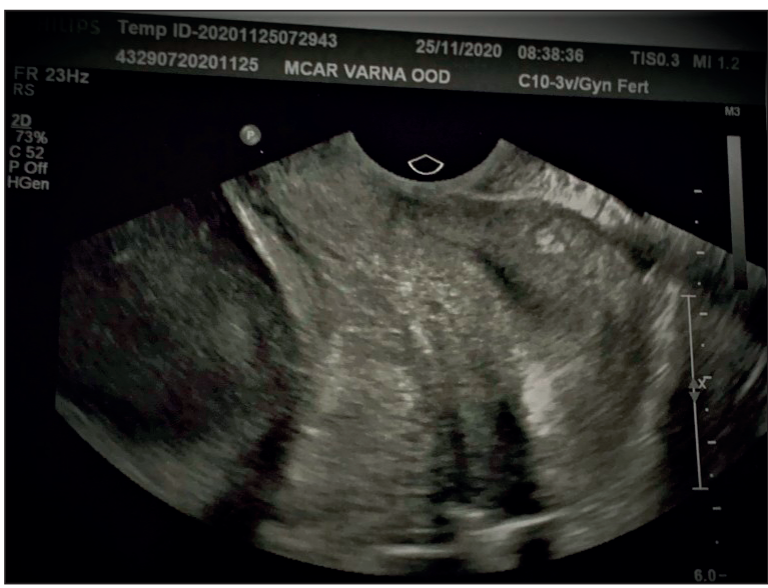

Figure 3. Ultrasonography - sagittal view of a retroverted uterus and a free fluid in the Douglas pouch with varying echogenicity.

abscess formation, because locally impaired immunity in the pelvic cavity makes the patient susceptible to infection ${ }^{10}$. Both the Royal College of Obstetricians and Gynaecologists and the European Society of Human Reproduction and Embryology recommend resection for endometriomas measuring $4 \mathrm{~cm}$ or larger, in order to reduce the risk of infection ${ }^{11,12}$. This topic is still debated in the field of reproductive medicine. Whether to perform a surgical removal of an endometriotic cyst before applying any assisted reproductive techniques or to put the patient at risk of possible complications is a question to which there are still no guidelines to answer ${ }^{13}$.

In our case, the diagnosis was easily made, mainly because of the typical clinical manifestation and ultrasonographic signs of ruptured ovarian abscess, in combination with the knowledge of pre-existing

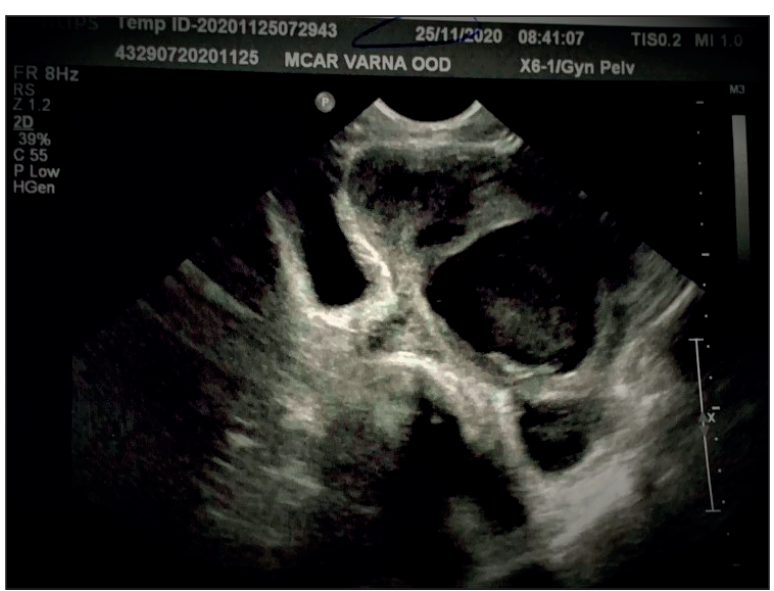

Figure 2. Ultrasonography - transverse plane of the right adnexa demonstrating varying sized cystic appearing structures consistent with tubal-ovarian abscess. The bladder is also visualized.

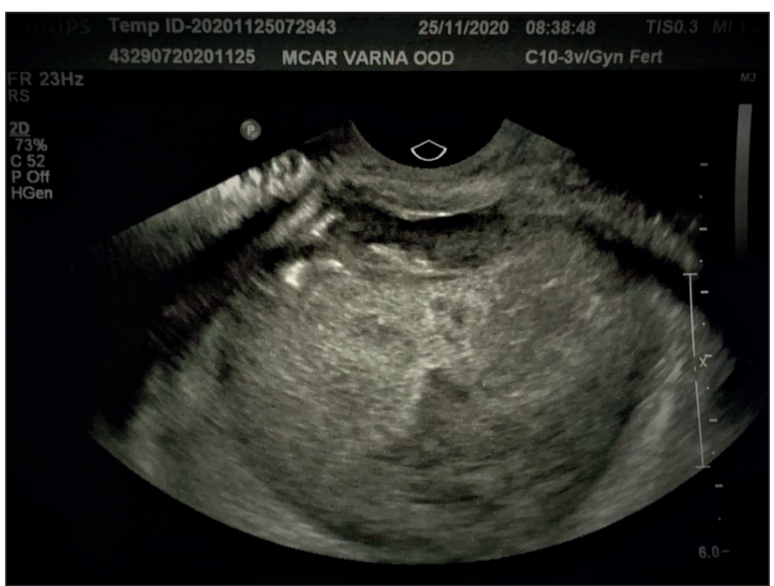

Figure 4. Ultrasonography - transverse plane of the uterus showing diffuse enlargement.

endometrioma and suspected puncture of the cyst during oocyte retrieval. In some similar cases described in the literature, the symptoms occur late, within months after $\mathrm{TVOR}^{14}$. In this peculiar case, the symptoms started one day after oocyte retrieval, but were probably diminished due to the prescribed prophylactic antibiotic treatment.

Treatment of pelvic abscess in women of reproductive age or during pregnancy is always difficult. The choice to avoid surgical treatment for preserving fertility or prolong pregnancy may lead to unwanted maternal and perinatal complication or even death ${ }^{15}$. The embryo transfer in our case was done 12 days before her admission in the hospital and since it was too early to diagnose a pregnancy, our decision was not influenced by assuring maturity and therefore better survival chances of the foetus, rather than saving the 
patient's life. After aggravation of patient's symptoms, we proceeded to surgical treatment of the ruptured abscess. Laparoscopy was the preferred route, because it has more advantages (shorter operation duration, shorter length of hospital stay, less bleeding) compared to laparotomy. The prompt decision of surgical management of the patient allowed us to be more conservative and keep the uterus and the left adnexa intact. We performed only right salpingo-oophorectomy with lavage and pelvic drain placement, which was removed within three days post-surgery. Unfortunately, the day after surgery moderate uterine bleeding occurred and pregnancy loss was proven with quantitative human chorion gonadotropin blood test.

\section{Conclusions}

Ovarian abscess may develop in women with endometrioma following assisted reproductive techniques. The condition, although rare, may complicate coexisting pregnancy, with the risks of spontaneous abortion or preterm labour and may also lead to severe complications for the woman. Thus, the prompt recognition of the condition is of great importance and, based on our experience, the immediate surgical treatment after symptoms aggravation despite the aggressive antibiotic therapy allows to be as conservative as possible in terms of fertility preservation.

\section{Author Contributions:}

N.T., E.K., V.I. were responsible for the diagnostic procedures, clinical diagnosis, and treatment decisions. T.K. performed the surgery. D.I. and N.T. wrote the manuscript. V.I. translated the abstract in French. All authors have read and agreed to the published version of the manuscript.

\section{Compliance with Ethics Requirements:}

"The authors have no conflict of interest relevant to this article"

"The authors declare that all the procedures and experiments of this study respect the ethical standards in the Helsinki Declaration of 1975, as revised in 2008, as well as the national law. Informed consent was obtained from the patient included in the study"

"No funding for this study"

\section{Acknowledgments}

None

\section{REFERENCES}

1. Brosens I. Endometriosis and the outcome of in vitro fertilization. Fertil Steril. 2004;81:1198-200.

2. Garcia-Velasco JA, Arici A. Surgery for the removal of endometriomas before in vitro fertilization does not increase implantation and pregnancy rates. Fertil Steril. 2004;81:1206.

3. Gibbons WE. Management of endometriosis in fertility patients. Fertil Steril. 2004;81:1204-5.

4. Somigliana E, Vercellini P, Vigano P, Ragni G, Crosignani PG. Should endometriomas be treated before IVF-ICSI cycles? Hum Reprod Update. 2006;12:57-64.

5. Garcia-Velasco JA, Mahutte NG, Corona J, et al. Removal of endometriomas before in vitro fertilization does not improve fertility outcomes: a matched, case-control study. Fertil Steril. 2004;81:1194-7

6. Verkauf BS. The incidence, symptoms, and signs of endometriosis in fertile and infertile women. J Fla Med Assoc. 1987;74:671-675.

7. Padilla SL. Ovarian abscess following puncture of an endometrioma during ultrasound-guided oocyte retrieval. Hum Reprod. 1993;8:1282-3

8. Moini A, Riazi K, Amid V, et al. Endometriosis may contribute to oocyte retrieval-induced pelvic inflammatory disease: report of eight cases. J Assist Reprod Genet. 2005;22:307-309.

9. Chen MJ, Yang JH, Yang YS, Ho HN. Increased occurrence of tubo-ovarian abscesses in women with stage III and IV endometriosis. Fertil Steril. 2004;82:498-9.

10. Matsunaga Y, Fukushima K, Nozaki M, et al. A case of pregnancy complicated by the development of a tubo-ovarian abscess following in vitro fertilization and embryo transfer. American Journal of Perinatology. 2003;20(06):277-282.

11. Leyland N, Casper R, Laberge P, Singh SS; SOGC. Endometriosis: diagnosis and management. J Obstet Gynaecol Can. 2010 Jul;32(7 Suppl 2):S1-32. PMID: 21545757.

12. Kennedy S, Bergqvist A, Chapron C, et al. ESHRE guidelines for the diagnosis and treatment of endometriosis. Hum Reprod. 2005;20:2698-2704.

13. Demirol A, Guven S, Baykal C, Gurgan T. Effect of endometrioma cystectomy on IVF outcome: a prospective randomized study. Reprod Biomed Online. 2006;12:639-43.

14. Han C, Wang C, Liu XJ, et al. In vitro fertilization complicated by rupture of tubo-ovarian abscess during pregnancy. Taiwan J Obstet Gynecol. 2015;54(5):612-6.

15. Khawaja N, Walsh T, Gleeson R, Geary MPP. Tubal abscess in pregnancy: A case report. Journal of Obstetrics and Gynaecology. 2005; 25(2):210. 